\title{
Adherence to American Association for the Study of Liver Diseases (AASLD) Guidelines and Predictors of Readmission in Cirrhotic Patients: A Single Center Experience
}

\author{
Maen M. Masadeh1 ${ }^{*}$, Ali Zaied1, Feroze Hussain'², Heidi Spratt ${ }^{3}$, Roger Soloway² \\ ${ }^{1}$ Department of Internal Medicine, University of Texas Medical Branch at Galveston, Galveston, USA \\ ${ }^{2}$ Department of Gastroenterology and Hepatology, University of Texas Medical Branch at Galveston, \\ Galveston, USA \\ ${ }^{3}$ Department of Biochemistry and Molecular Biology, University of Texas Medical Branch at Galveston, \\ Galveston, USA \\ Email: maen masadeh@hotmail.com
}

Received 13 May 2015; accepted 28 May 2015; published 4 June 2015

Copyright (C) 2015 by authors and OALib.

This work is licensed under the Creative Commons Attribution International License (CC BY). http://creativecommons.org/licenses/by/4.0/

(c) (i) Open Access

\section{Abstract}

Introduction: Liver cirrhosis (LC) is a major cause of mortality and morbidity in the United States. American Association for the Study of Liver Disease (AASLD) has developed guidelines for the management of patients with LC. Methods: We conducted a one-year retrospective chart review study of patients admitted with LC related complication. Our primary outcome was adherence to AASLD guidelines for the management of variceal bleeding (VB), fluid overload and hepatocellular carcinoma (HCC) screening, and secondary outcome was re-admission rate within 30 days of discharge. Results: A total of 139 patients were reviewed. Majority were males (65\%) and Caucasians (65\%). The admission indication was mainly for fluid overload (39\%), hepatic encephalopathy (36\%) and VB (27\%). The one-month readmission rate was $31 \%$, of those $47 \%$ and $37 \%$ were admitted for hepatic encephalopathy and fluid overload respectively. Periodic screening for HCC was done in only $40 \%$ of patients. Ninety-five percent of patients admitted with VB received PPI, octreotride and antibiotics. Diet education was only documented in $9 \%$ of patients on discharge. Significant predictors for 30-day readmission included high MELD score, elevated creatinine, and taking diuretics/lactulose before hospitalization in addition to shorter length of stay. Conclusion: Compliance with AASLD guidelines was optimal for patients with VB. Further optimization is required for HCC screening and patient education. Multiple factors play a role in readmissions for patients with cirrhosis; this model helps to identify patients at risk for readmission and opens an area for quality improvement measures to avoid unnecessary hospitalizations.

\footnotetext{
${ }^{*}$ Corresponding author.

How to cite this paper: Masadeh, M.M., Zaied, A., Hussain, F., Spratt, H. and Soloway, R. (2015) Adherence to American Association for the Study of Liver Diseases (AASLD) Guidelines and Predictors of Readmission in Cirrhotic Patients: A Single Center Experience. Open Access Library Journal, 2: e1593. http://dx.doi.org/10.4236/oalib.1101593
} 


\title{
Keywords
}

\section{Liver Cirrhosis, Readmission, Hepatic Encephalopathy, Ascites}

\author{
Subject Areas: Gastroenterology \& Hepatology
}

\section{Introduction}

Liver cirrhosis (LC) is a major cause of mortality and morbidity in the United States. More than 100,000 patients are discharged each year with chronic liver disease and cirrhosis as the first-listed diagnosis [1]. It is the 12th leading cause of death in adults in the United States. It results in 31,903 deaths per year with 10.3 deaths per 100,000 population [2].

Liver fibrosis results from the perpetuation of the normal wound healing response resulting in an abnormal continuation of fibrogenesis (connective tissue production and deposition). Fibrosis progresses at variable rates depending on the cause of liver disease, environmental and host factors [3]. Cirrhosis is frequently indolent, asymptomatic and unsuspected until complications of liver disease present. However, initial clinical presentation of patients with decompensated cirrhosis is still common and is characterized by the presence of dramatic and life-threatening complications, such as variceal hemorrhage, ascites, spontaneous bacterial peritonitis, or hepatic encephalopathy [3]. Radiological diagnosis of liver cirrhosis can be challenging as ultrasonography, computerized tomography and magnetic resonance imaging are not sensitive to detect cirrhosis, and final diagnosis still relies on histology. However, their specificity is high when an obvious cause is present and imaging reveals an inhomogeous hepatic texture or surface, rarefied hepatic central vein, an enlarged caudate lobe, splenomegaly or collateral veins [4].

Ascites is the most common major complication of cirrhosis; other major complications include: hepatic encephalopathy (HE) and variceal hemorrhage [5]. The mainstays of treatment of LC patients with ascites include patient education regarding dietary sodium restriction (2000 mg/day [88 mmol/day]) and oral diuretics [6] [7].

Variceal bleeding is considered the most dangerous and life threatening consequence of portal hypertension [8]. Management of suspected acute variceal bleeding includes: adequate resuscitation with airway protection \& acceptable volume support, somatostatin analogues (to be continued for 3 - 5 days once varices are proven), short-term (5 - 7 days) prophylactic antibiotic therapy and Esophagogastroduodenoscopy as soon as possible to confirm the diagnosis and implement endoscopic therapy [9].

Primary liver cancer (hepatocellular carcinoma, HCC) is a serious late complication of chronic hepatic injury, particularly in cirrhosis due to Hepatitis B Virus, Hepatitis C Virus, and hemochromatosis. The incidence of HCC has increased by 70\% in the United States over the past 20 years, particularly among younger patients [10], Biannual screening for HCC by alpha fetoprotein testing and ultrasound has shown to reduce mortality [11].

Hospital readmissions among patients with decompensated liver cirrhosis are common, costly, moderately predictable —in some cases possibly preventable—and independently associated with mortality [12]. Multiple factors are thought to play a role like nursing, system, physician and not less significant patients' factors. Managing patients with liver cirrhosis decompensated by variceal bleeding, ascites or hepatic encephalopathy by following AASLD guidelines would optimize care and subsequently lead to significant decrease in morbidity and mortality [8] [10] [11] [13].

The aim of this study was to evaluate the adherence to AASLD guidelines at a tertiary teaching center and to assess for predictors of re-hospitalization within 30 days after discharge.

\section{Methods}

We conducted a retrospective chart review for patients with decompensated LC admitted to University of Texas Medical Branch (UTMB) at Galveston from June 2012 to June 2013. Cases were identified using International Classification of Diseases, 9th revision (ICD-9) codes: 571.2 (alcoholic cirrhosis), 571.5 (non-alcoholic cirrhosis), 572.3 (portal hypertension), 572.2 (hepatic encephalopathy), 456.0 to 456.2 (esophageal varices with and without bleeding), 789.5 (ascites), 789.59 (nonmalignant ascites), 567.23 (spontaneous bacterial peritonitis), and 572.4 (hepatorenal syndrome). Electronic medical records were reviewed by the authors MM and AZ. Liver cirrhosis 
was confirmedeither by histology if liver biopsy was done and showed grade 4 fibrosis, or by imaging if ultrasound (US), Computed Tomography (CT) or Magnetic Resonance Imaging (MRI) showed typical features of advanced liver disease; recanalization of umbilical vein, nodularity of the liver, presence of vascular collaterals and reversal of portal blood flow. We included patients with LC who were admitted for: fluid overload, HE, variceal bleeding, hepatorenal syndrome, SBP or abdominal pain. We excluded patients who were admitted for any other reasons including infection other than SBP, patients who left the hospital against medical advice and hospice patients. For patients with multiple admissions, the latest admission that meets the inclusion criteria was included.

\subsection{Outcome}

Our primary outcome was adherence to AASLD guidelines for the management of variceal hemorrhage, fluid overload and hepatocellular carcinoma screening. Adherence to AASLD guidelines for the management of variceal hemorrhage was defined as: use of proton pump inhibitor, somatostatin analogues and antibiotic therapy for SBP prophylaxis at the time of admission. Adherence to AASLD guidelines for the management of fluid overload was defined as: use of diuretics, salt restriction and patient education. Adherence to AASLD guidelines for the screening of HCC was defined as: Liver ultrasound or CT scan every 6 - 12 months. Our secondary outcome was re-admission rate within 30 days of discharge with the exclusion of elective admissions.

\subsection{Date Collection}

Study data were collected and managed using REDCap electronic data capture tools hosted at UTMB [14]. REDCap (Research Electronic Data Capture) is a secure, web-based application designed to support data capture for research studies, providing 1) an intuitive interface for validated data entry; 2) audit trails for tracking data manipulation and export procedures; 3 ) automated export procedures for seamless data downloads to common statistical packages; and 4) procedures for importing data from external sources.

The institutional review board (IRB) approved our study. Variables collected include demographics (age, sex, and race), etiology of cirrhosis, and presence of hepatocellular carcinoma, comorbid conditions and reason for hospitalization. Laboratory values of interest included white blood cell count, platelet count, sodium level, creatinine level, albumin level, total bilirubin, and international normalized ratioon admission. Model for End-stage Liver Disease (MELD) score and Child's Pugh score were both calculated. Data regarding whether the patient required intensive level of care or was readmitted within 30 days of discharge was also gathered.

\subsection{Statistical Analysis}

Descriptive statistics was conducted using STATA software for the primary outcome. For the secondary outcome Multivariate Adaptive Regression Splines (MARS) model was developed on $80 \%$ of the data (training), and tested on the remaining $20 \%$ (testing). MARS is a nonparametric regression procedure that seeks to create a classification model based on piecewise linear regressions. This algorithm has the ability to search through a large number of candidate predictor variables to determine those most relevant to the classification model. We used five variables as input, and created a model with the ability to correctly predict hospital readmission with an $81.2 \%$ success rate in the training population and a $72.73 \%$ success rate in the testing population based on these five variables.

\section{Results}

A total of 139 patients met the inclusion criteria. The baseline characteristics of these patients are summarized in Table 1. The majority of patients were males (64.8\%) and Caucasians (65.5\%). The most common etiologies of LC were alcoholic liver disease (61.2\%), hepatitis C virus infection (49.6\%) and fatty liver disease (7.9\%) while other etiologies like autoimmune hepatitis, Hemochromatosis and primary biliary cirrhosis made less than $10 \%$. The indication for admission included fluid overload (38.9\%), HE (36.0\%), variceal bleeding (26.6\%), abdominal pain (11.5\%), SBP (5.0\%) and coagulopathy (2.9\%).

One third of patients required Intensive Care Unit (ICU) level of care. A hepatologist, either as a consult service or as a primary service, was involved in $84.2 \%$ of patients' care. Only $31 \%$ of patients followed up with hepatology or gastroenterology clinic within 1 month after discharge. Physical exam findings and laboratory values on presentation are shown in Table 2 and Table 3 respectively. 
Table 1. Baseline characteristics of the patients.

\begin{tabular}{|c|c|}
\hline & $\mathrm{N}=139$ \\
\hline Age-year & $54.6 \pm 9.9$ \\
\hline Male sex-n. (\%) & $90(64.8)$ \\
\hline \multicolumn{2}{|l|}{ Race-n. (\%) } \\
\hline African American & $16(11.5)$ \\
\hline Caucasian & $91(65.5)$ \\
\hline Hispanic & $32(23.0)$ \\
\hline \multicolumn{2}{|l|}{ Etiology of cirrhosis-n. $(\%)^{*}$} \\
\hline $\mathrm{HCV}$ & $69(49.6)$ \\
\hline Alcohol & $86(61.2)$ \\
\hline HBV & $2(1.4)$ \\
\hline NAFLD & $11(7.9)$ \\
\hline Autoimmune & $6(4.3)$ \\
\hline Other $^{\dagger}$ & $9(6.5)$ \\
\hline \multicolumn{2}{|l|}{ Reason for admission $-\mathrm{n} .(\%)^{*}$} \\
\hline Variceal Bleeding & 37 (26.6) \\
\hline Ascites & $54(38.9)$ \\
\hline Hepatic Encephalopathy & $50(36.0)$ \\
\hline Coagulopathy & $4(2.9)$ \\
\hline SBP & $7(5.0)$ \\
\hline Abdominal Pain & $16(11.5)$ \\
\hline Other $^{\ddagger}$ & $12(8.6)$ \\
\hline Hepatologist involved in patients' care-n. $(\%)^{\S}$ & $117(84.2)$ \\
\hline 30 day re-admission—n. (\%) & $43(31.0)$ \\
\hline \multicolumn{2}{|l|}{ Procedures-n. $(\%)^{*}$} \\
\hline Paracentesis & $54(38.9)$ \\
\hline EGD & $45(32.3)$ \\
\hline Colonoscopy & $5(3.6)$ \\
\hline TIPS & $6(4.3)$ \\
\hline Liver Biopsy & $3(2.2)$ \\
\hline ICU level of care-n. (\%) & $46(33.1)$ \\
\hline Mortality—n. (\%) & $12(8.6)$ \\
\hline \multicolumn{2}{|l|}{ Comorbidity—n. (\%) } \\
\hline Heart Disease & $16(11.5)$ \\
\hline Diabetes & 27 (19.4) \\
\hline Renal Failure ${ }^{\pi}$ & 44 (31.7) \\
\hline COPD & $5(3.6)$ \\
\hline HIV & $2(1.4)$ \\
\hline Malignancy' & $35(25.2)$ \\
\hline
\end{tabular}

"Patients may have more than one reason to be admitted, etiology of cirrhosis or procedure performed. ${ }^{\dagger}$ Include: Hemochromatosis (1), Wilson Disease (0), PBC (2), PSC (1), Alpha 1 Anti Trypsin Deficiency (1), Secondary Liver Cancer (1) and Cryptogenic (3). ${ }^{*}$ Include: AKI, Alcoholic hepatitis, Cholangitis, HCC, Hepatic hydrothorax, Jaundice, Umbilical hernia. ${ }^{8}$ Either as admitted to liver service or as a consult service when admitted to non-liver service. "Includes: AKI (30), CKD (9) and ESRD (5). 'Includes HCC (22) and other (13). Abbreviations: HCV: Hepatitis C Virus; HBV: Hepatitis B Virus; NAFLD: Non Alcoholic Fatty Liver Disease; PBC: Primary Biliary Cirrhosis; PSC: Primary Sclerosing Cholangitis; AKI: Acute Kidney Injury; CKD: Chronic Kidney Disease; ESRD: End Stage Renal Disease; HCC: Hepatocellular Cancer; EGD: Esophagogastroduodenoscopy; TIPS: Transjugular Intrahepatic Portosystemic Shunt; ICU: Intensive Care Unit; COPD: Chronic Obstructive Pulmonary Disease; HIV: Human Immunodeficiency Virus 
Table 2. Physical examination findings of the patients.

\begin{tabular}{cc}
\hline & $\mathrm{N}=139$ \\
\hline Systolic Blood Pressure $>100 \mathrm{~mm} \mathrm{Hg}-\mathrm{n}$. (\%) & $122(87.8)$ \\
Heart Rate, rate/min-n. (\%) & $4(2.9)$ \\
$<50$ & $4(2.9)$ \\
$50-59$ & $87(62.6)$ \\
$60-99$ & $44(31.4)$ \\
Mental Status-n. (\%) & $99(71.2)$ \\
Alert & $20(14.4)$ \\
Disoriented & $13(9.4)$ \\
Stuporous & $7(5.0)$ \\
Comatose & $24(17.3)$ \\
Flapping Tremor-n. (\%) & $81(58.3)$ \\
Ascites—n. (\%) & $68(49.0)$
\end{tabular}

Table 3. Laboratory results of the patients.

\begin{tabular}{cc}
\hline Hgb, g/dL & Value $( \pm$ SD $)$ \\
\hline WBC count, $10^{6}$ cells/dL & $10.3( \pm 2.3)$ \\
Platelet count, $10^{3}$ cells/ $\mu \mathrm{L}$ & $8.0( \pm 5.2)$ \\
INR & $120( \pm 86)$ \\
Albumin, g/dL & $1.8( \pm 2.0)$ \\
Total Bilirubin, U/L & $2.8( \pm 0.6)$ \\
Sodium, $\mathrm{mEq} / \mathrm{L}$ & $4.9( \pm 6.8)$ \\
Potassium, $\mathrm{mEq} / \mathrm{L}$ & $136( \pm 6)$ \\
Creatinine, $\mathrm{mg} / \mathrm{dL}$ & $4.3( \pm 0.8)$ \\
MELD score & $1.4( \pm 1.4)$ \\
Child Pugh Score & $18( \pm 8)$ \\
\hline
\end{tabular}

Abbreviations: SD: Standard Deviation; Hgb: Hemoglobin; WBC: White Blood Cell; INR: International Normalized Ratio; MELD: Model for End Stage Liver Disease.

For patients admitted with fluid overload $(n=54), 31.7 \%$ of them were diuretics naïve, and dose adjustment on discharge was done in $57.6 \%$. Eighty seven percent of them were discharged on low sodium diet but patient education was only documented in $7.4 \%$.

For patients admitted with variceal bleeding $(n=37)$, prior to admission screening esophagogastroduodenoscopy was done in $74.8 \%$ of them. Proton pump inhibitors, octreotide and SBP prophylaxis were given on admissionto $94.6 \%$ of patients.

Hepatocellular cancer screening modalities included liver ultrasound (manufacturer General Electric) (56.1\%), CT (manufacturer General Electric) with triple phase contrast (62.6\%) and MRI (manufacturer General Electric) 
of the liver (6.5\%). For patients who are eligible for HCC screening, interval screening every 6 to 12 months was done in only $40.3 \%$.Primary study outcomes are summarized in Table 4.

Thirty-day readmission rate was $31.0 \%$, of those $47 \%$ and $37 \%$ were admitted for HE and fluid overload respectively. Significant predictors for 30-day readmission included high MELD score and elevated creatinine. These results are similar to results found by Volk et al. [12]. Also, Patients who were taking diuretics and/or lactulose before hospitalization were found more likely to be readmitted. Average length of stay (LOS) was 5.2 days, and patients with a shorter LOS were more likely to be readmitted. Etiology of cirrhosis, reason for admission and Child Pugh score were not found to be important predictors. The Multivariate adaptive regression splines (MARS) model predicted 30-day readmission with an area under the receiver operating characteristic curve of 0.87 and 0.74 in the training and testing data respectively with a y-value of 0.6 (Figure 1).

\section{Discussion}

Patients with chronic liver disease presenting with acute decompensation are often seriously ill. The AASLD proposed guidelines for the management of such events like variceal bleeding [9] [13] and ascites and fluid over load [15]. Guidelines were also proposed for HCC screening [16]. In this study we evaluated the adherence to standard of care for these patients. Despite the presence of evidence for best practice for patients with ascites and fluid overload with educating the patients about their diet being a cornerstone in avoiding decompensation [6] [7] [15], this was only provided to $7.4 \%$ of the patients. Moreover screening for HCC per guidelines was only

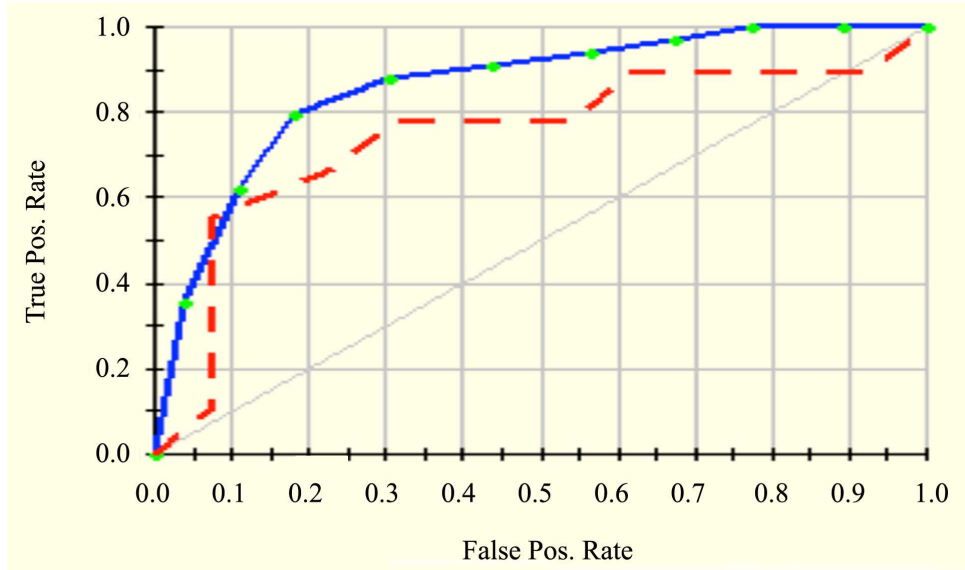

Figure 1. Multivariate adaptive regression splines (MARS) Model. Blue line training data, Red line testing data.

Table 4. Study primary outcomes.

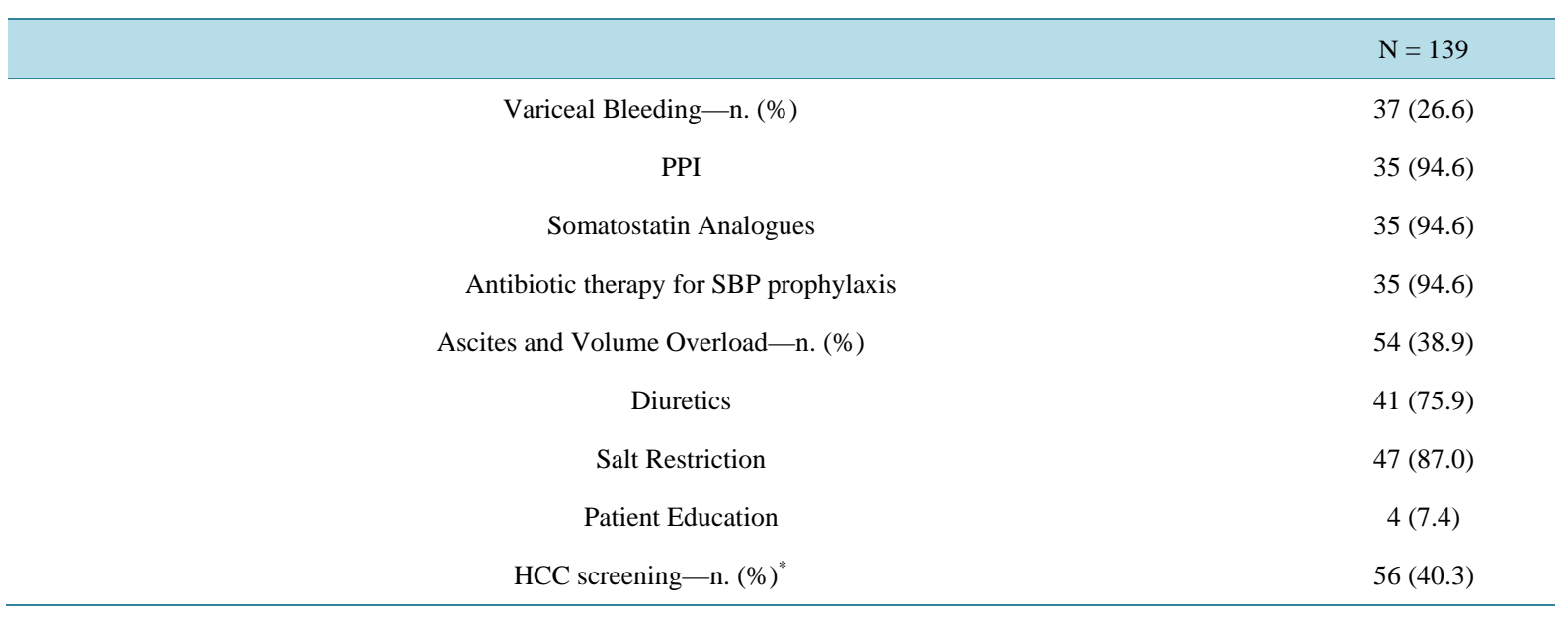

*Every 6 to 12 month; Abbreviations: PPI: Proton Pump Inhibitor; SBP: Spontaneous Bacterial Peritonitis. 
40.3\%. Acute medical management with proton pump inhibitors, somatostatin analogues and antibiotics for SBP prophylaxis for patients admitted with bleeding varices was optimal (94.6\%).

The 30-day readmission rate was 31\%. It is lower than what Volk et al. reported (37\%) [12], but higher than the study conducted by Berman et al. (20\%) [17]. There are no current studies that show the national average of cirrhosis 30-day readmission rate.

This study shows another predictive role of MELD score; patients with a higher MELD score were found more likely to be readmitted; this was also reported by Berman et al. [17].

Johnson et al. [8] has described several interventions to control some of the factors that may lead to rehospitalization like providing an order set for variceal bleeding and patients' education for patients with ascites.In his prospective cohort study, using an admission order set forvariceal bleeding and providing educational sessions for medical practitioners were associated with a statistically significant lower number of 30-day readmissions rate $(41 \%$ vs. $13 \%$; $\mathrm{P}<0.001)$. However, there was no significant decrease in length of hospital stay

Shu et al. [18] reported that longer LOS was associated with a higher rate of 30-day readmission, our study showed the opposite where patients with shorter length of stay were found to be more likely readmitted within 30 days of discharge. This might indicate that these patients need optimal inpatient management before discharge.

Cirrhosis is a complex disease with multiple complications that are overwhelming for patients and their care providers. Our current episodic and uncoordinated system of healthcare delivery is poorly structured for such patients with chronic disease [19]. This study points out some breaches in managing these patients; and it may open a way for certain interventions to help optimize the care of LC patient.

The limitations of our study include: 1) It's retrospective; 2) It was done in a single center; 3) The sample size is relatively small. Also re-admissions could have happened to other hospitals in the area.

\section{Conclusion}

In summary, compliance with AASLD guidelines was optimal for patients with variceal bleeding. Further optimization is required for HCC screening and patient education for ascites. We believe that this study will add to the literature in several ways. First, it helps identifying gaps in treating patients with LC. Second, it outlines predictors of 30-day readmission that can help identifying patients that might benefit from post discharge care. Further prospective studies are needed to better identify and possibly implement quality measures that might improve patients' outcome and avoid unnecessary hospitalizations.

\section{References}

[1] CDC/NCHS. National Hospital Discharge Survey (2010) Detailed Diagnosis and Procedure Tables; Number of FirstListed Diagnoses for Discharges from Short-Stay Hospitals, by ICD-9-CM Code, Sex, Age, and Geographic Region.

[2] Murphy, S., Xu, J. and Kochanek, K. (2013) Deaths: Final Data for 2010. National Vital Statistics Reports, 61, 1-118.

[3] Schuppan, D. and Afdhal, N.H. (2008) Liver Cirrhosis. The Lancet, 371, 838-851. http://dx.doi.org/10.1016/S0140-6736(08)60383-9

[4] Martínez-Noguera, A., Montserrat, E., Torrubia, S. and Villalba, J. (2002) Doppler in Hepatic Cirrhosis and Chronic Hepatitis. Seminars in Ultrasound, CT and MRI, 23, 19-36. http://dx.doi.org/10.1016/S0887-2171(02)90027-2

[5] Gines, P., Quintero, E., Arroyo, V., et al. (1987) Compensated Cirrhosis: Natural History and Prognostic Factors. Hepatology (Baltimore, Md), 7, 122-128. http://dx.doi.org/10.1002/hep.1840070124

[6] Runyon, B.A. (1994) Care of Patients with Ascites. The New England Journal of Medicine, 330, 337-342. http://dx.doi.org/10.1056/NEJM199402033300508

[7] Runyon, B.A. (2015) Sleisenger and Fordtran’s Gastrointestinal and Liver Disease. 10th Edition, Saunders, Philadelphia, PA, Chapter 93, 1553.e5-1576.e5.

[8] Johnson, E.A., Spier, B.J., Leff, J.A., Lucey, M.R. and Said, A. (2011) Optimising the Care of Patients with Cirrhosis and Gastrointestinal Haemorrhage: A Quality Improvement Study. Alimentary Pharmacology \& Therapeutics, 34, $76-$ 82. http://dx.doi.org/10.1111/j.1365-2036.2011.04692.x

[9] Garcia-Tsao, G. and Bosch, J. (2010) Management of Varices and Variceal Hemorrhage in Cirrhosis. The New England Journal of Medicine, 362, 823-832. http://dx.doi.org/10.1056/NEJMra0901512

[10] El-Serag, H.B. and Mason, A.C. (1999) Rising Incidence of Hepatocellular Carcinoma in the United States. The New England Journal of Medicine, 340, 745-750. http://dx.doi.org/10.1056/NEJM199903113401001 
[11] Zhang, B.H., Yang, B.H. and Tang, Z.Y. (2004) Randomized Controlled Trial of Screening for Hepatocellular Carcinoma. Journal of Cancer Research and Clinical Oncology, 130, 417-422. http://dx.doi.org/10.1007/s00432-004-0552-0

[12] Volk, M.L., Tocco, R.S., Bazick, J., Rakoski, M.O. and Lok, A.S. (2012) Hospital Readmissions among Patients with Decompensated Cirrhosis. The American Journal of Gastroenterology, 107, 247-252. http://dx.doi.org/10.1038/ajg.2011.314

[13] Garcia-Tsao, G., Sanyal, A.J., Grace, N.D. and Carey, W. (2007) Prevention and Management of Gastroesophageal Varices and Variceal Hemorrhage in Cirrhosis. Hepatology (Baltimore, Md), 46, 922-938. http://dx.doi.org/10.1002/hep.21907

[14] Harris, P.A., Taylor, R., Thielke, R., Payne, J., Gonzalez, N. and Conde, J.G. (2009) Research Electronic Data Capture (REDCap) - A Metadata-Driven Methodology and Workflow Process for Providing Translational Research Informatics Support. Journal of Biomedical Informatics, 42, 377-381. http://dx.doi.org/10.1016/j.jbi.2008.08.010

[15] Runyon, B.A. (2009) Management of Adult Patients with Ascites Due to Cirrhosis: An Update. Hepatology (Baltimore, Md), 49, 2087-2107. http://dx.doi.org/10.1002/hep.22853

[16] Bruix, J. and Sherman, M., American Association for the Study of Liver D (2011) Management of Hepatocellular Carcinoma: An Update. Hepatology (Baltimore, Md), 53, 1020-1022. http://dx.doi.org/10.1002/hep.24199

[17] Berman, K., Tandra, S., Forssell, K., et al. (2011) Incidence and Predictors of 30-Day Readmission among Patients Hospitalized for Advanced Liver Disease. Clinical Gastroenterology and Hepatology: The Official Clinical Practice Journal of the American Gastroenterological Association, 9, 254-259. http://dx.doi.org/10.1016/j.cgh.2010.10.035

[18] Shu, C.C., Lin, Y.F., Hsu, N.C. and Ko, W.J. (2012) Risk Factors for 30-Day Readmission in General Medical Patients Admitted from the Emergency Department: A Single Centre Study. Internal Medicine Journal, 42, 677-682. http://dx.doi.org/10.1111/j.1445-5994.2011.02562.x

[19] Volk, M.L., Piette, J.D., Singal, A.S. and Lok, A.S. (2010) Chronic Disease Management for Patients with Cirrhosis. Gastroenterology, 139, 14e1-16e1.

\section{Abbreviations}

LC: Liver cirrhosis

AASLD: American Association for the Study of Liver Disease

VB: Variceal Bleeding

HCC: Hepatocellular Carcinoma

HE: Hepatic Encephalopathy

SBP: Spontaneous Bacterial peritonitis

MELD: Model for End Stage Liver Disease

MARS: Multivariate Adaptive Regression Splines

LOS: Length of Stay

CT: Computed Tomography

MRI: Magnetic Resonance Imaging 